\title{
Performance Improvement of Deflection Routing in Optical Burst Switching Networks
}

\author{
SuKyoung Lee ${ }^{1}$, Kotikalapudi Sriram ${ }^{1}$, HyunSook Kim² ${ }^{2}$, and JooSeok Song ${ }^{2}$ \\ 1 National Institute of Standards and Technology, \\ 100 Bureau Drive, Stop 8920, Gaithersburg, MD 20899, USA. \\ 2 Dept. of Computer Science, Yonsei University, Seoul, Korea
}

\begin{abstract}
In Optical Burst Switching (OBS) networks, when contention occurs at an intermediate switch, two or more bursts that are in contention can be lost because a forwarding path reservation is not made for a burst until a control message for the burst arrives. While deflection routing protocol is proposed as one of the contention resolution techniques, there has been no appropriate deflection routing algorithm to find an alternate route. In this paper, we propose a novel deflection routing algorithm to compute alternate routes with better performance as compared to other known techniques. This algorithm deflects contending bursts using a path that is based on minimization of a performance measure that combines distance and blocking due to contention. We will show, through simulation results, that there is an improvement in terms of loss with increased network throughput.
\end{abstract}

\section{Introduction}

For building IP over WDM network, we have several alternatives. One promising approach is optical burst switching [1]. One of challenging issues in application of burst switching to optical domain is the resolution of contentions that results from multiple incoming bursts that are directed to the same output port. In an optical burst switch, various techniques designed to resolve contentions include optical buffering, wavelength conversion and deflection routing. Over the other techniques, deflection routing gains an advantage that buffer capacity is very limited to maintain a reasonable level of data losses while routing the contending bursts to an output port other than the intended output port. However, before attempting to implement deflection routing in OBS networks, the details of deflection routing have to be investigated.

Some most recent studies about deflection routing can be found in [2]-[5]. In [2], the performance of deflection routing is examined in OBS networks based on Just-Enough-Time (JET). The authors of [3] and [4] demonstrated via simulation test that the blocking probability is improved when deflection routing is used as a contention resolution. On the other hand, the authors of [5] introduced how deflection routing can be applied to the self-routing address scheme. However, they do not explain how to route an alternate path i.e. which constraints could be applied to the selection of an Alternate Route (AR). 
In this paper, we introduce a Contention-based Deflection Routing (CDR) algorithm for computing Alternate Routes (ARs) in a way that avoids burst blocking due to contention. The objective of this paper is to formulate the deflection routing problems in mathematical form to motivate more work towards the development of efficient solution techniques. The main purpose of this algorithm is to recompute an optical path that is already established, so that the AR can be placed into the network being expected a burst would experience less blocking on the AR than on the shortest alternate path usually adopted in other known deflection routing works [2]-[5]. The proposed technique can also be applied to pre-configuration of primary routes with improved performance given that the burst intensity of each node is periodically measured by the network management system.

The rest of this paper is organized as follows. Section 2 describes CDR algorithm in detail. In Section 3, we present the simulation model used and performance of the proposed CDR is evaluated via computer simulation. Finally, we conclude the paper in Section 4.

\section{Deflection Routing Algorithm}

In OBS, a control packet is sent first to set up a connection by reserving an appropriate amount of bandwidth and configuring the switches along a path, followed by a data burst without waiting for an acknowledgement for the connection establishment. A delay referring to offset time or Fiber Delay Line (FDL) has been proposed to bring this form of reservation to fruition. The offset time allows for the control packet to be processed at each node while the burst is buffered electronically at the source; thus no FDLs are necessary at the intermediate nodes to delay the burst while the packet is being processed.

The proposed CDR can be applied to both styles of reservation now that CDR is not the mechanism of how to reserve the wavelength but how to select the alternative path. However, in consideration of the fact that the required FDL technology is not mature enough for it to be implemented in practice, it may be more beneficial to the current carriers that CDR is implemented on the basis of offset-based scheme since it maintains the most state information for the bursts and therefore allows for more flexible deflection routing.

In an OBS network, the deflection routing functions implemented in each switch automate the AR setups when a control packet encounters a congested node over the primary path. However, the switches have only local information about the network resources, especially the contention status. These local routing decisions for the ARs made in edge nodes may result in a degraded global network performance on the long run. As a solution for this, the proposed CDR algorithm is to perform periodical global re-optimization of ARs based on the most recent information of contention status. Even though this re-optimization is periodical, we mean that it is not performed not too frequently (i.e. daily or weekly). It is also possible for the re-optimization to be performed on demand. 
To formulate the CDR problem, the network topology, a set of attributes pertaining to the resources and the constraints in the network are defined. The demands that are to be routed through ARs in the network are described by a set of attributes as well. Then, the problem is to find an optimal AR minimizing a cost function which explicitly accounts for the contention rate as well as the burst hop distance. The aforementioned CDR problem can be formulated as follows: Consider a physical network represented by a graph $G(N, L)$ where $N$ is the set of nodes and $L$ the set of links (i.e. fibers) connecting the nodes. It is assumed that each link between nodes $i$ and $j$, has $W_{i j}$ wavelengths with the same capacity of $C$. At each node $n,(n=1, \ldots,|N|)$, the number of transmitters and receivers are defined as $P_{n}^{(t)}$ and $P_{n}^{(r)}$, respectively. If a node $n$ has the number, $P_{n}$ of ports, clearly, at most $\sum_{n} P_{n}$ wavelengths are needed to realize any possible virtual topology.

Let $\Lambda$ be the set of traffic demands belonging to the loss-sensitive service class between a pair of edge nodes, where $\lambda_{i j}^{s d} \in \Lambda$ represents the arrival rate of bursts from source $s$ to destination $d$ that flows over an virtual link between node $i$ and node $j$, and where $\lambda_{s_{k} d_{k}}$ denotes the average flow of bursts associated with the $k^{\text {th }}$ traffic demand requesting wavelength. Let $D=\left\{D_{i j}\right\}$ be the distance matrix from node $i$ to node $j$ that means a propagation delay from node $i$ to node $j$ $(i \neq j)$. As the cost of contention from node $i$ to node $j(i \neq j)$, we introduce a constant $b_{i j}$ to denote burst blocking rate that is collected periodically from the network.

In the CDR problem formulation, the variable that needs to be determined is $x_{i j} . x_{i j}$ is a binary variable associated with link $(i, j)$ to indicate whether $\left(x_{i j}=1\right)$ or not $\left(x_{i j}=0\right)$ the link $(i, j)$ is established over AR. The problem is to find an AR from the congested node to the destination, for which the burst blocking rate and the distance are minimized. Then, this problem can be formulated for the $k^{\text {th }}$ traffic flow as follows:

$$
\text { Minimize } g_{d} \sum_{i, j} x_{i j} D_{i j}+g_{b}\left[\log _{10}\left[1-\prod_{i, j}\left(1-x_{i j} b_{i j}\right)\right]\right.
$$

where $g_{d}$, and $g_{b}$ denote the weights for delay and blocking, respectively.

such that

$$
\begin{gathered}
\sum_{\forall j \in N} x_{i j} \leq P_{i}^{(t)}, \sum_{\forall i \in N} x_{i j} \leq P_{j}^{(r)} \\
\lambda_{i j}=x_{i j} \sum_{s, d} \lambda_{i j}^{s d}+x_{i j} \lambda_{s_{k} d_{k}} \quad \forall i, j \in N \\
\lambda_{i j}^{s_{k} d_{k}} \in\left\{0, \lambda_{s_{k} d_{k}}\right\} \quad \forall i, j \in N \\
\sum_{j} x_{i j}-\sum_{j} x_{j i}=\left\{\begin{array}{c}
1, i=s_{k} \\
-1, i=d_{k} \\
0, \text { otherwise }
\end{array} \quad \forall s, d, i \in N\right. \\
\lambda_{i j} \leq W_{i j} C \quad \forall i, j \in N
\end{gathered}
$$


Eq. 1 defines the objective function. This objective function is a weighted sum of the end-to-end burst blocking rate and the distance for the route. To decrease the computational complexity, we can express the above objective function, Eq. 1 as

$$
\text { Miminize } \quad g_{d} \sum_{i, j} x_{i j} D_{i j}+g_{b} \sum_{i, j} x_{i j} \log _{10} b_{i j},
$$

where as the burst contention rate, $b_{i j}$, the real data can be used that has been collected into deflection routing information base. Constraint 2 ensures that the number of lightpaths originating from and terminating at a switch is not more than the switch's out-degree and in-degree, respectively. There are some constraints (3-6) related to the traffic flow on virtual topology for each link $(i, j)$. Constraint 3 states that the total flow on the simplex link from node $i$ to node $j$ is expressed as the superposition of the existing traffic (i.e. bursts) and the new burst associated with the link. Since we are setting up an AR for the optical bursts coming from a specific traffic flow, the bursts of the demand $\lambda_{s_{k} d_{k}}$ are not segmented at any congested node in the network. Thus, constraint 4 defines that the traffic demand $\lambda_{s_{k} d_{k}}$ is routed from node $i$ to $j$ on a single AR. Constraint 5 formulates flow conservation at each node. The traffic flowing into a node should be equal to that flowing out of the node for any node other than the source and destinations for each traffic flow $k$. Constraint 6 assures that traffic flowing through a link cannot exceed the total link capacity.

We know that the objective function in Eq. 7 is more of a practical value than one involving distance alone. It includes Quality of Service (QoS) requirements regarding loss as well as distance. This objective function can be easily generalized to the case of multiple Classes of Service (CoS), where bursts of different $\mathrm{CoS}$ may have different QoS requirements regarding loss. The disparate CoS and their required QoS can be reflected into the routing decision by having different weights associated with each in our objective function. In the end, the AR would be set up according to the values of the $x_{i j}$ determined from the above integer linear programming formulation.

As mentioned above, the optimization algorithm described in this section can be performed offline or online. In the former approach, multiple fixed ARs are considered when a contention occurs. Thus, each node in the network is required to maintain a deflection routing table that contains an ordered list of a number of fixed ARs to each destination node. In the on-demand CDR, the AR from a congested node to a destination node is chosen dynamically, depending on the current network state. The on-demand CDR method will require more computations and a longer response time than CDR based on pre-computed alternate routes and lookup-table. But the on-demand CDR approach is more flexible and would result in better resource utilization and performance than the latter approach.

For offset-based scheme [6], one of representative reservation styles in an OBS network, the burst will not arrive successfully to its destination over the AR computed by CDR without extra offset time or buffered delay. When deflection routing is performed due to a contention at an intermediate node, the offset time 
on the AR is different from(usually, longer than) that on the primary path. One solution to this problem is to render sufficient extra offset time to each burst while the other is making the control packet reserve FDL buffer to delay the burst. Even though the above problem is resolved, it may happen that the too much increased distance on AR cause longer delay than expected offset time or buffering time. Thus, if $t_{o, c}$ denotes a maximum limit of offset time for service class $c$, that consists of basic offset time and extra offset time, another constraint for offset time is defined as

$$
\sum_{i, j} x_{i j} D_{i j} \leq t_{o, c} \quad \forall i, j
$$

\section{Performance Evaluation}

In order to evaluate the effectiveness of CDR technique, simulation tests are conducted under two schemes: CDR algorithm and Shortest Path-based Deflection Routing (SPDR) algorithm which has been generally used in other deflection routing works [3]-[5]. Our simulation is run on JET [6] which is one of offsetbased schemes.

In our simulation, we assume that each fiber link is composed of 6 wavelengths and its bandwidth is $1 \mathrm{Gbps}$. The burst sources were individually simulated with the on/off model, where the on and off periods were varied as ( $\alpha^{-1}=120 \mathrm{~ms}$, $\left.\beta^{-1}=880 \mathrm{~ms}\right)$ and $\left(\alpha^{-1}=300 \mathrm{~ms}, \beta^{-1}=700 \mathrm{~ms}\right)$

The tests are carried out using two particular network scenarios: a simple network topology with five nodes as figure 1 in [3] and 14-node NSFNET in Fig. 1 as a larger topology. Over NSFNET topology, as soon as five ingress-egress node pairs are chosen randomly, optical bursts are generated at the ingress nodes of a optical burst network domain.

In Fig. 1, some bursts whose source and destination are CA1 and NJ, respectively, experience a contention at UT node on the primary path (CA1-UT-MINJ). As an example in our simulation, the deflection routing table at the node

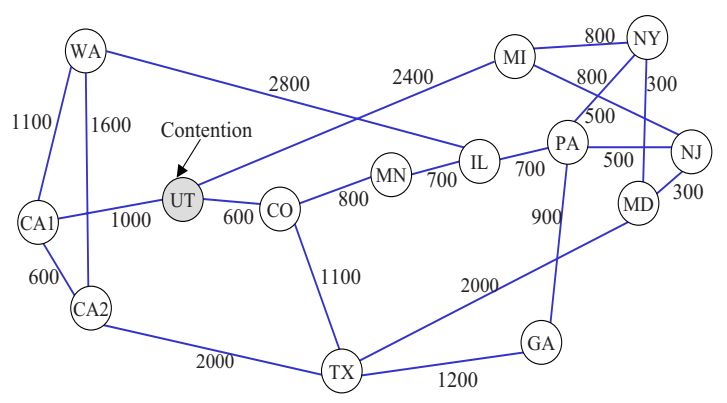

Fig. 1. Simulation network topology 
Table 1. Burst drop rate and overall throughput improvement(Mbps) over five-nodes topology

\begin{tabular}{c|c|c|c}
\hline \hline $\begin{array}{c}\text { Burst size } \\
\text { (Bytes) }\end{array}$ & \multicolumn{2}{|c|}{ Burst drop rate } & Throughput \\
\cline { 2 - 3 } & CDR & SPDR & improvement \\
\hline \hline 576 & $1.14 \times 10^{-5}$ & $3.44 \times 10^{-4}$ & 2.39 \\
2304 & $1.53 \times 10^{-5}$ & $3.36 \times 10^{-4}$ & 8.49 \\
4608 & $1.62 \times 10^{-5}$ & $3.40 \times 10^{-4}$ & 17.27 \\
\hline \hline
\end{tabular}

lists the (UT-CO-MN-IL-PA-NJ) and (UT-CO-TX-MD-NJ) as alternate candidate paths. Of these, (UT-CO-MN-IL-PA-NJ) is the shortest alternate path from UT to NJ. However, the CDR scheme can very well select (UT-CO-TXMD-NJ) as the preferred alternate path if that happens to be the only one that meets the requirement on burst blocking as well as distance. The focus of our performance evaluation is on data loss rate caused by contention. A burst will be dropped if both the primary and deflection paths are blocked. The data loss rate for the entire network is found by calculating the average of the burst drops for each source-destination pair. Thus, we first show that the data loss rate is indeed reduced by CDR technique in comparison to SPDR.

Table 1 shows the results of simulation performed with 3 different burst sizes as in [1] when offered traffic load is 0.6. As can be seen from this table, CDR scheme drops smaller number of bursts than SPDR with improved throughput.

Figs. 2 and 3 show simulation results comparing the burst blocking or loss rate for our CDR method with the SPDR method. The SPDR algorithm simply picks the shortest path alternate route available from the deflection routing table, whereas with the CDR scheme the alternate path selection is based on

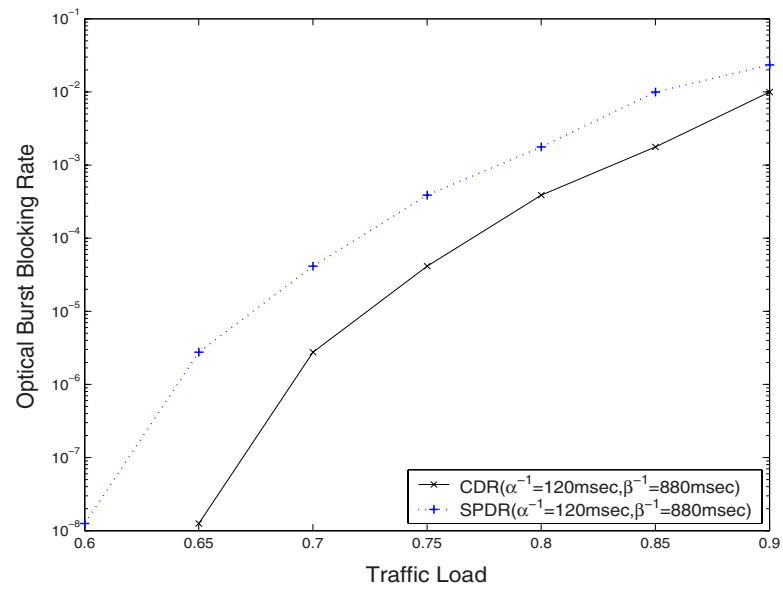

Fig. 2. Burst blocking rate for CDR and SPDR without FDL when activity is 0.12 


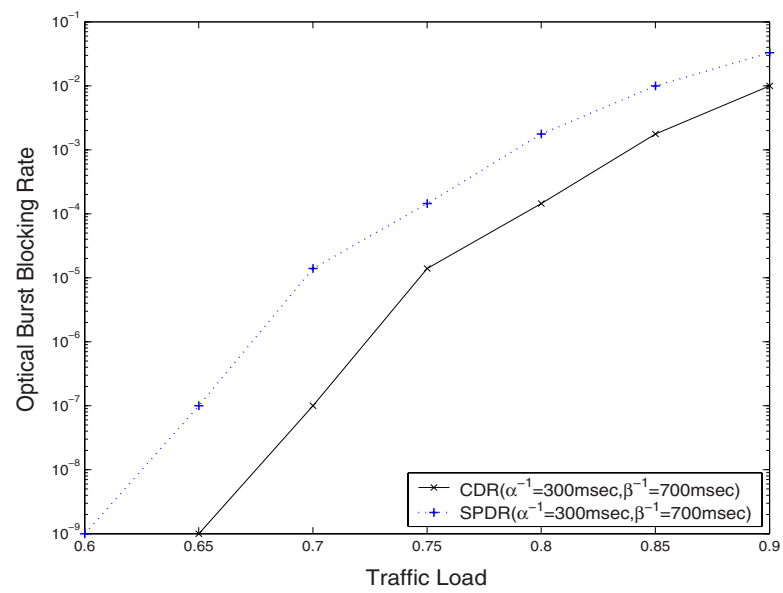

Fig. 3. Burst blocking rate for CDR and SPDR without FDL when activity is 0.3

minimizing a composite performance measure consisting of the alternate path distance as well as burst blocking along that path. For typical operating load values up to 0.75 , the CDR algorithm improves burst blocking by more than an order of magnitude as compared to SPDR in the test cases that we have studied through simulation runs.

Fig. 4 shows how much the overall network throughput was improved by CDR algorithm, compared to SPDR algorithm. The throughput values shown are the values averaged during the entire simulation time. In this figure, as the traffic load increases and more bursts contend on the shortest AR, the more

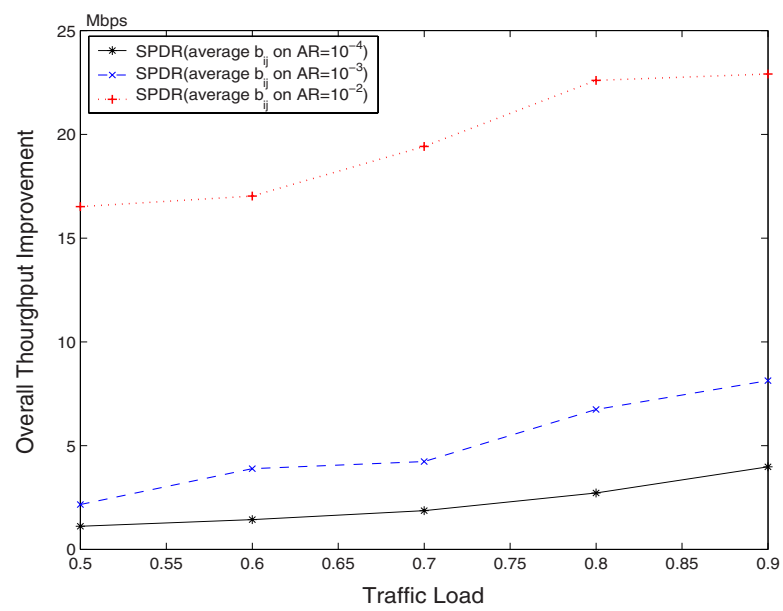

Fig. 4. Overall network throughput improvement by CDR when compared to SPDR 
data is accommodated into a network by CDR than SPDR. We can see that throughput performance is improved under CDR compared with SPDR.

For average end-to-end delay the difference between both schemes is around $1.6 \%$. As we expected, the average delay increases for CDR because the bursts have more deflections to arrive the destination. Therefore, we know that CDR algorithm can satisfy the QoS requirement of loss-sensitive traffic. Especially, in the case that the contention rate gets higher on the AR selected by SPDR algorithm, the better performance can be achieved by CDR algorithm in terms of loss and throughput.

\section{Conclusion}

We note that when deflection routing is used as a contention resolution, it is important to design ARs in an optimized fashion to minimize burst blocking. Our simulation results have shown that the proposed CDR scheme enables lower burst blocking rate and higher network throughput as compared to the SPDR scheme. This is because CDR selects ARs that are not necessarily shortest path but have the lowest burst blocking rate, based on periodically updated measurement data.

An area of future work can be to extend the CDR algorithm with scheduling policies that consider traffic types with different QoS requirements in OBS networks.

\section{References}

1. H.M. Chaskar, S. Verma, and R. Ravikanth, "Robust Transport of IP Traffic over WDM Using Optical Burst Switching", Optical Network Magazine, vol. 3, no. 4, pp. 47-60, Jul/Aug. 2002.

2. C-F. Hsu, T-L Liu and F-F Huang, "Performance Analysis of Deflection Routing in Optical Burst-Switched Networks", IEEE INFOCOM'02, 2002.

3. X. Wang, H. Morikawa, and T. Aoyama, "Burst Optical Deflection Routing Protocol for Wavelength Routing WDM Networks", Optical Networks Magazine, November/December 2002, pp. 12-19.

4. S. Kim, N. Kim, and M. Kang, "Contention Resolution for Optical Burst Switching Networks Using Alternative Routing", IEEE ICC'02, vol. 5, pp. 2678-2681, NewYork, USA, Apr/May. 2002.

5. C.Y. Li et al, "Deflection Routing in Slotted Self-routing Networks with Arbitrary Topology", IEEE ICC'02, vol.5, pp. 2781-2785, NewYork, USA, Apr/May. 2002.

6. C. Qiao, and M. Yoo, "Optical Burst Switching (OBS) - A New Paradigm for an Optical Internet," Journal of High Speed Networks, vol. 8, no. 1, pp. 69-84, 1999. 